\title{
Appendix 2 Interview series with Catalan and Scottish politicians
}

Between 18 September 2002 and 21 November 2002, 28 interviews were conducted in Scotland. The 25 interviews in Catalonia were conducted between 6 February 2003 and 26 March 2003. Interview partners consist of Catalan and Scottish politicians (and some journalistic and academic observers) from a wide range of institutions and positions (see list in table below). Interviews were all conducted by the author. They were semi-structured with variations depending on the position of interviewees, yet leaving also enough leeway to follow individual points. They lasted between half an hour and two hours, with an average length of one hour and were conducted in English and Spanish (plus two in German). All but seven of them were recorded and fully transcribed.

Varying degrees of secrecy and anonymity were agreed with different interview partners and for different parts of the interviews. They ranged from full disclosure and direct attribution, over passages attributed to a particular office or function (e.g. Labour MP or PP party official), to complete off-the-record remarks. As a consequence interview statements are used and reproduced in various different forms: as pure background information, as non-attributable statements (referred to in the text with interview number in brackets), as statements attributed to a particular office, or to a particular person.

The following complete list of interviews shows the names of interviewees, the places and dates at which they were conducted, plus the party affiliation and the positions and offices interview partners were holding at the time of the interview. 
Table A2.1 List of interviews

\begin{tabular}{|c|c|c|c|c|}
\hline Name & Place & Date & Party & $\begin{array}{l}\text { Main position (s) } \\
\text { held at time of } \\
\text { interview }\end{array}$ \\
\hline \multicolumn{5}{|l|}{ Catalonia } \\
\hline Pujol, Jordi & Barcelona & 24.03 .2003 & $\mathrm{CiU} / \mathrm{CDC}$ & $\begin{array}{l}\text { MCP; President } \\
\text { of Catalonia }\end{array}$ \\
\hline $\begin{array}{l}\text { Riera i Bou, } \\
\text { Marcel }\end{array}$ & Barcelona & 12.02 .2003 & $\mathrm{CiU} / \mathrm{CDC}$ & MCP \\
\hline Campuzano i & Barcelona & 21.03 .2003 & $\mathrm{CiU} / \mathrm{CDC}$ & Spanish \\
\hline Canadès, Carles & & & & $\begin{array}{l}\text { Congress } \\
\text { member }\end{array}$ \\
\hline Molins, Joaquim & Barcelona & 14.03 .2003 & $\mathrm{CiU} / \mathrm{CDC}$ & $\begin{array}{l}\text { Former } \\
\text { politician }\end{array}$ \\
\hline $\begin{array}{l}\text { Vidal i Gayolà, } \\
\text { Joan }\end{array}$ & Barcelona & 18.03 .2003 & $\mathrm{CiU} / \mathrm{CDC}$ & $\begin{array}{l}\text { Senior civil } \\
\text { servant, Catalan } \\
\text { administration }\end{array}$ \\
\hline Grifells, Salvador & Barcelona & 15.03 .2003 & $\mathrm{CiU} / \mathrm{CDC}$ & $\begin{array}{l}\text { Communication } \\
\text { officer, CDC }\end{array}$ \\
\hline $\begin{array}{l}\text { Casas i Bedós, } \\
\text { Jordi }\end{array}$ & Barcelona & 13.03 .2003 & $\mathrm{CiU} / \mathrm{UDC}$ & $\begin{array}{l}\text { MCP; speaker, } \\
\text { UDC }\end{array}$ \\
\hline $\begin{array}{l}\text { Puigdomènech i } \\
\text { Cantó, Carles }\end{array}$ & Barcelona & 13.03 .2003 & $\mathrm{CiU} / \mathrm{UDC}$ & MCP \\
\hline Rigol y Roig, Joan & nBarcelona & 17.03 .2003 & $\mathrm{CiU} / \mathrm{UDC}$ & $\begin{array}{l}\text { MCP; President } \\
\text { of Catalan } \\
\text { Parliament }\end{array}$ \\
\hline $\begin{array}{l}\text { Huguet i Biosca, } \\
\text { Josep }\end{array}$ & Barcelona & 05.02 .2003 & ERC & $\begin{array}{l}\text { MCP; speaker } \\
\text { of parl. group; } \\
\text { deputy general } \\
\text { secretary, ERC }\end{array}$ \\
\hline $\begin{array}{l}\text { Vendrell i Segura, } \\
\text { Xavier }\end{array}$ & Barcelona & 11.03 .2003 & ERC & $\begin{array}{l}\text { MCP; national } \\
\text { secretary } \\
\text { organisation and } \\
\text { finance, ERC }\end{array}$ \\
\hline $\begin{array}{l}\text { Ribó i Masso, } \\
\text { Rafael }\end{array}$ & Barcelona & 17.02 .2003 & $\mathrm{ICV}$ & $\begin{array}{l}\text { MCP; speaker } \\
\text { parl. group; } \\
\text { professor of } \\
\text { politics, UAB }\end{array}$ \\
\hline $\begin{array}{l}\text { Mejías Sánchez, } \\
\text { Caridad }\end{array}$ & Barcelona & 26.03 .2003 & PP & MCP \\
\hline $\begin{array}{l}\text { Sirera i Bell, } \\
\text { Daniel }\end{array}$ & Barcelona & 17.03 .2003 & PP & $\begin{array}{l}\text { MCP; deputy } \\
\text { speaker parl. } \\
\text { group; deputy } \\
\text { general } \\
\text { secretary, PP }\end{array}$ \\
\hline
\end{tabular}


Table A2.1 (continued)

\begin{tabular}{|c|c|c|c|c|}
\hline Name & Place & Date & Party & $\begin{array}{l}\text { Main position (s) } \\
\text { beld at time of } \\
\text { interview }\end{array}$ \\
\hline $\begin{array}{l}\text { Clotas i Cierco, } \\
\text { Higini }\end{array}$ & Barcelona & 12.03 .2003 & PSC & $\begin{array}{l}\text { MCP; vice } \\
\text { president } \\
\text { Catalan } \\
\text { Parliament }\end{array}$ \\
\hline $\begin{array}{l}\text { Nadal i Farreras, } \\
\text { Joacquim }\end{array}$ & Barcelona & 12.02 .2003 & PSC & $\begin{array}{l}\text { MCP; speaker } \\
\text { of parliamentary } \\
\text { group }\end{array}$ \\
\hline $\begin{array}{l}\text { Roma i Cunill, } \\
\text { Joan }\end{array}$ & Barcelona & 12.03 .2003 & PSC & MCP; mayor \\
\hline $\begin{array}{l}\text { Simó i Huguet, } \\
\text { Josep }\end{array}$ & Barcelona & 13.03 .2003 & PSC & MCP \\
\hline Inglada, Xavier & Barcelona & 25.03 .2003 & PSC & $\begin{array}{l}\text { Chief executive, } \\
\text { PSC }\end{array}$ \\
\hline $\begin{array}{l}\text { Espasa i Oliver, } \\
\text { Ramon }\end{array}$ & Barcelona & 13.03 .2003 & $\mathrm{PSC} / \mathrm{CpC}$ & $\begin{array}{l}\text { MCP PSC- } \\
\text { CpC, formerly } \\
\text { PSUC/ICV }\end{array}$ \\
\hline Valles i & Barcelona & 12.02 .2003 & $\mathrm{PSC} / \mathrm{CpC}$ & MCP; leader \\
\hline Casadevall, Josep & & & & $\begin{array}{l}\text { CpC; professor } \\
\text { of politics, UAB }\end{array}$ \\
\hline Argelaguet, Jordi & Barcelona & 10.02 .2003 & & $\begin{array}{l}\text { Lecturer in } \\
\text { politics, UAB }\end{array}$ \\
\hline $\begin{array}{l}\text { Calvet i Crespo, } \\
\text { Jordi }\end{array}$ & Barcelona & 13.02 .2003 & & $\begin{array}{l}\text { Lecturer in } \\
\text { politics, UB }\end{array}$ \\
\hline $\begin{array}{l}\text { Matas i Dalmases, } \\
\text { Jordi }\end{array}$ & Barcelona & 20.02 .2003 & & $\begin{array}{l}\text { Professor of } \\
\text { politics, UB }\end{array}$ \\
\hline Pau, Francesc & Barcelona & 05.02 .2003 & & $\begin{array}{l}\text { Senior staff, } \\
\text { Catalan } \\
\text { Parliament }\end{array}$ \\
\hline $\begin{array}{l}\text { Scotland } \\
\text { Douglas- } \\
\text { Hamilton, James }\end{array}$ & Edinburgh & 30.09 .2002 & Con & MSP \\
\hline Fraser, Murdo & Edinburgh & 19.11 .2002 & Con & MSP \\
\hline Johnston, Alex & Edinburgh & 02.10 .2002 & Con & $\begin{array}{l}\text { MSP; } \\
\text { Conservative } \\
\text { group business } \\
\text { manager }\end{array}$ \\
\hline Tosh, Murray & Edinburgh & 14.11 .2002 & Con & $\begin{array}{l}\text { MSP; deputy } \\
\text { presiding } \\
\text { officer, Scottish } \\
\text { Parliament }\end{array}$ \\
\hline
\end{tabular}


Table A2.1 (continued)

\begin{tabular}{|c|c|c|c|c|}
\hline Name & Place & Date & Party & $\begin{array}{l}\text { Main position(s) } \\
\text { beld at time of } \\
\text { interview }\end{array}$ \\
\hline Mitchell, David & Edinburgh & 30.09 .2002 & Con & $\begin{array}{l}\text { Chairman, } \\
\text { Scottish } \\
\text { Conservative } \\
\text { and Unionist } \\
\text { Party }\end{array}$ \\
\hline Rodger, Alan & Edinburgh & 30.09 .2002 & Con & $\begin{array}{l}\text { Research } \\
\text { assistant, } \\
\text { Scottish } \\
\text { Conservative } \\
\text { and Unionist }\end{array}$ \\
\hline Hughes, Janice & Edinburgh & 12.11 .2002 & Labour & MSP \\
\hline McAllion, John & Edinburgh & 08.10 .2002 & Labour & MSP \\
\hline McNeil, Duncan & Edinburgh & 09.10 .2002 & Labour & $\begin{array}{l}\text { MSP; Labour } \\
\text { group whip }\end{array}$ \\
\hline Reid, George & Edinburgh & 12.11 .2002 & Labour & $\begin{array}{l}\text { MSP; deputy } \\
\text { presiding } \\
\text { officer, Scottish } \\
\text { Parliament }\end{array}$ \\
\hline Galbraith, Sam & Glasgow & 18.11 .2002 & Labour & $\begin{array}{l}\text { Retired Labour } \\
\text { politician }\end{array}$ \\
\hline Welsh, Ian & Glasgow & 18.09 .2002 & Labour & Former MSP \\
\hline Lazarowicz, Mark & Edinburgh & 15.11 .2002 & Labour & $\mathrm{MP}$ \\
\hline Matheson, Murdo & Glasgow & 21.11 .2002 & Labour & $\begin{array}{l}\text { party researcher, } \\
\text { Labour }\end{array}$ \\
\hline Rumbles, Mike & Edinburgh & 06.11 .2002 & Lib-Dem & MSP \\
\hline Stone, Jamie & Edinburgh & 09.10 .2002 & Lib-Dem & MSP \\
\hline Wallace, Jim & Edinburgh & 03.10 .2002 & Lib-Dem & $\begin{array}{l}\text { MSP; deputy } \\
\text { First Minister, } \\
\text { Scottish } \\
\text { Executive; } \\
\text { leader of } \\
\text { Scottish Liberal } \\
\text { Democrats }\end{array}$ \\
\hline Barrie, Derek & Edinburgh & 19.11 .2002 & Lib-Dem & $\begin{array}{l}\text { Chief of staff, } \\
\text { Scottish Liberal } \\
\text { Democrats }\end{array}$ \\
\hline McArthur, Liam & Edinburgh & 20.09 .2002 & Lib-Dem & $\begin{array}{l}\text { Special adviser } \\
\text { to deputy first } \\
\text { minister }\end{array}$ \\
\hline Robison, Shona & Edinburgh & 01.10 .2002 & SNP & $\begin{array}{l}\text { MSP; secretary } \\
\text { of SNP group }\end{array}$ \\
\hline
\end{tabular}


Table A2.1 (continued)

\begin{tabular}{|c|c|c|c|c|}
\hline Name & Place & Date & Party & $\begin{array}{l}\text { Main position(s) } \\
\text { held at time of } \\
\text { interview }\end{array}$ \\
\hline Hosie, Stewart & Inverness & 27.09 .2002 & SNP & $\begin{array}{l}\text { National } \\
\text { secretary, SNP }\end{array}$ \\
\hline Maxwell, Stephen & Edinburgh & 24.09 .2002 & SNP & $\begin{array}{l}\text { Senior officer, } \\
\text { Scottish Council } \\
\text { for Voluntary } \\
\text { Org. }\end{array}$ \\
\hline $\begin{array}{l}\text { Ross-Williams, } \\
\text { Capre }\end{array}$ & Edinburgh & 01.10 .2002 & SNP & $\begin{array}{l}\text { Westminster } \\
\text { researcher; } \\
\text { Holyrood } \\
\text { candidate }\end{array}$ \\
\hline Wishart, Peter & Inverness & 28.09 .2002 & SNP & $\mathrm{MP}$ \\
\hline Grice, Paul & Edinburgh & 13.11 .2002 & & $\begin{array}{l}\text { Chief executive/ } \\
\text { chief clerk, } \\
\text { Scottish } \\
\text { Parliament }\end{array}$ \\
\hline Wright, Kenyon & Edinburgh & 05.11 .2002 & & $\begin{array}{l}\text { Executive chair } \\
\text { of Scottish } \\
\text { Constitutional } \\
\text { Convention }\end{array}$ \\
\hline Young, Ben & Edinburgh & 20.11 .2002 & & $\begin{array}{l}\text { Researcher } \\
\text { Scottish Civic } \\
\text { Forum }\end{array}$ \\
\hline Mitchell, James & Glasgow & 25.09 .2002 & & $\begin{array}{l}\text { Professor } \\
\text { of politics, } \\
\text { Strathclyde } \\
\text { University }\end{array}$ \\
\hline
\end{tabular}

